\title{
Synthèse de l'atelier "Corruption et bureaucratie"
}

Report of workshop "Corruption and Bureaucracy"

\section{Giorgio Blundo et Walter E.A. van Beek}

\section{OpenEdition}

\section{Journals}

Édition électronique

URL : http://journals.openedition.org/apad/173

DOI : 10.4000/apad.173

ISSN : 1950-6929

Éditeur

LIT Verlag

\section{Édition imprimée}

Date de publication : 1 décembre 2002

Référence électronique

Giorgio Blundo et Walter E.A. van Beek, « Synthèse de l'atelier "Corruption et bureaucratie" », Bulletin de I'APAD [En ligne], 23-24 | 2002, mis en ligne le 15 décembre 2006, consulté le 22 septembre 2020.

URL : http://journals.openedition.org/apad/173 ; DOI : https://doi.org/10.4000/apad.173

Ce document a été généré automatiquement le 22 septembre 2020

Bulletin de l'APAD 


\title{
Synthèse de l'atelier "Corruption et bureaucratie"
}

\author{
Report of workshop "Corruption and Bureaucracy"
}

Giorgio Blundo et Walter E.A. van Beek

\section{NOTE DE L'ÉDITEUR}

Rapporteur : Giorgio Blundo. Discutant : Walter E.A. van Beek

1 L'atelier s'est articulé autour de huit contributions, qui fournissent, par la diversité des terrains et des secteurs étudiés, un panorama non exhaustif mais suffisamment éclairant quant aux multiples facettes du phénomène corruptif en Afrique subsaharienne : la gestion officieuse des communes urbaines (B. Beridogo) et le racket des usagers dans les services publics, en particulier la police routière et la douane (M. Djiré), au Mali ; la corruption dans les relations entre le personnel médical et les usagers de la santé au Cameroun (J-F. Médard) et en Côte d'Ivoire (O. Gadou); les détournements au sein d'un programme de coopération bilatérale au Sénégal (M. Mathieu) ; le système complexe des pots-de-vin et des commissions au sein des marchés publics au Tchad (G. Mahoundonodji); les relations ambiguës entre la presse privée et gouvernementale et l'État ghanéen (J. Hast y) ; et enfin une contribution qui analyse l'évolution dans les perceptions de la fonction publique de la part des jeunes diplômés béninois (A. Konen). Même si cette étude ne traite pas directement des pratiques corruptrices, elle montre comment l'administration étatique demeure, aux yeux de la jeunesse, un lieu stratégique pour l'inscription dans des réseaux pouvant ouvrir la voie à des ressources (bourses de formation à l'étranger) et plus en général à des carrières internationales.

2 Au lieu de résumer le contenu de chaque papier et de détailler les discussions qui ont suivi chaque présentation, j'ai opté pour un traitement transversal et comparatif des grandes questions qui ont émergé au cours de l'atelier.

Questions de méthode 
3 La corruption est un objet difficile à saisir pour le chercheur : d'une part, elle est souvent occultée, dissimulée (au Mali on la désigne par l'expression "les choses de la nuit"), notamment lorsqu'il s'agit d"'affaires" portant sur des sommes importantes (les marchés publics pétroliers décrits par Mahoundonodji), ou quand les pratiques corruptrices entrent en profonde contradiction avec l'éthique d'un corps professionnel (c'est le cas du secteur privé confessionnel de la santé, présenté par Médard) ou d'une institution (la coopération italienne, étudiée par Mathieu). D'autre part, plusieurs intervenants ont souligné l'extrême visibilité, voire banalisation, des pratiques quotidiennes de "petite corruption". Le risque est alors, pour l'observateur extérieur, de caractériser en tant que délits des actes qui sont légitimes d'un point de vue émique, étant profondément noyés dans un flux plus large de rapports sociaux culturellement acceptés et encouragés (échange de faveurs et de dons, règles de bienséance et de politesse, formes de protection et de patronage, comme le rappelle Van Beek). Lorsqu'elle est observable par le chercheur, la "corruption" demeure souvent inaperçue comme telle par les acteurs qui la pratiquent. Pour éviter l'impasse ethnocentrique, une! piste pourrait être celle d'explorer la sémiologie populaire de la corruption: restituer, dans leur contexte d'énonciation et d'expression, les mots et les gestes qui désignent et entourent les actes corruptifs. Plusieurs papiers présentés dans' l'atelier ont de fait évoqué le vocabulaire émique des pratiques politico-administratives illicites.

Il devient donc clair que les travaux discutés dans l'atelier ne se sont pas limités à la méthode classique de l'observation participante (dont on peut même questionner la pertinence pour le cas de la corruption). Certains ont mêlé des observations (tantôt relevant du vécu personnel et présentées sur le mode anecdotique, tantôt issues d'une inscription professionnelle dans un secteur particulier) à des entretiens informels ou formels; d'autres ont aussi eu recours à l'analyse d'articles de presse et de données quantitatives. La contribution de Mathieu représente en revanche le seul exemple de véritable étude de cas, construite, telle une enquête policière, par le croisement de données documentaires, de visites sur les lieux et d'entretiens.

5 Il est apparu aussi que travailler sur la corruption nécessite une connaissance approfondie des processus et des règlements formels qui régissent les différents services administratifs. C'est la seule voie pour apprécier - loin de toute velléité normative ou réformiste - l'écart entre normes formelles et pratiques quotidiennes, et pour déterminer comment les systèmes corruptifs produisent eux aussi des normes, généralement tacites et accessibles seulement aux initiés. Les acteurs de la corruption possèdent en effet leur propre étiquette, qu'il convient d'étudier pour chercher de comprendre, de l'intérieur, le fonctionnement de ce monde opaque, appréhendé le plus souvent à travers le seul point de vue des "victimes".

6 Enfin, plusieurs participants ont souligné que le thème de l'atelier incite, par son ubiquité, à un traitement de type comparatif: au sein d'un pays (entre secteurs différents et entre acteurs institutionnels au sein d'un même secteur, comme l'ont fait Médard pour la santé et Hasty pour la presse) ou entre pays (comparaison intra-africaine autour des formes de la petite corruption ou, en revanche, comparaison entre l'Afrique et d'autres continents en prenant comme point de départ la grande corruption).

Les mécanismes de la corruption

7 On remarque une assez grande uniformité dans les analyses des mécanismes de la corruption. La petite corruption, sans conteste la mieux représentée dans les 
contributions de l'atelier, apparaît comme une pratique de survie parmi d'autres, dans un contexte caractérisé par des fortes contraintes économiques et par la réduction des réseaux de solidarité traditionnels. Dans les pays étudiés, les mêmes tendances semblent favoriser la généralisation des pratiques corruptrices :

- ONG et projets de développement occupent aussi une place spécifique et importante dans les configurations foncières observées, qui peut relever dans certains cas de l' intermédiation et du courtage politico-institutionnel, comme dans les contributions présentés par Aïssatou Faye et Wiebe Nauta. Ils peuvent aussi exercer une influence directe sur les politiques nationales et le mode de fonctionnement des services étatiques, à l'exemple de la réforme sénégalaise de la politique forestière (Boutinot).

- des services publics discrédités, dysfonctionnels (c'est-à-dire considérés comme tels par les usagers et les fonctionnaires eux-mêmes) et orientés plus vers la satisfaction des intérêts des agents que des usagers ;

- des organigrammes officieux et informels opérationnels, face à des organigrammes officiels n'ayant de validité que "sur le papier" ; des budgets de fonctionnement insuffisants ;

- une fonction publique considérée comme un lieu d'accaparement de ressources et d'opportunités et en voie de privatisation informelle;

- une forte personnalisation dans l'accès à l'administration;

- une manipulation constante de règles et des normes en vigueur.

Quant aux pratiques décrites, elles prennent des formes différentes, selon les lieux et les pouvoirs de négociation des acteurs. On passe ainsi de l'extorsion (Djiré) à des formes de transaction (qui oscillent entre le cadeau de remerciement, comme le montre par exemple Médard pour l'univers sanitaire, le cadeau "anticipateur", comme le soli que reçoivent les journalistes ghanéens quand ils se rendent dans les ministères, selon Hast y , et la commission classique dans la passation d'un marché public, décrite par Mahoundonodji), à travers des pratiques d'appropriation privative de ressources publiques: détournement et vente de médicaments (Gadou et Médard), gestion privative des caisses municipales (Beridogo), etc.

Les représentations sociales de la corruption

Plusieurs papiers convergent en soulignant la grande ambivalence qui frappe les représentations locales des actes de corruption. La corruption est autant décriée que pratiquée au quotidien. Si, d'une part, l'opposition classique entre la figure du fonctionnaire corrompu et celle de l'usager victime ne résiste pas aux observations empiriques, d'autre part il faut envisager les conditions profondes de cette ambivalence entre discours et pratiques. Une piste s'entrevoit, celle de la recherche contextualisée des seuils à partir desquels s'opère le passage à l'acte corruptif ou se construit une perception contraire ou favorable à la corruption. Car ce phénomène relève de modes de légitimation et/ou de justification émiques, dont il faut repérer les registres, tout en évitant de les confondre avec les causes "objectives" de l'émergence de la corruption.

La lutte contre la corruption

Même si les contributions présentées demeurent essentiellement plus descriptives et analytiques que prescriptives, le débat qui a suivi a abordé également la réflexion sur les politiques de lutte. Si ce thème est cher aux chantres de la "bonne gouvernance", qu'ils soient nationaux ou internationaux, étatiques ou non-gouvernementaux, on ne peut pas passer sous silence que la corruption se constitue de plus en plus, en Afrique, comme un véritable problème de société, débattu dans les villages et véhiculé par la rumeur, bien avant d'être décrié dans les journaux. 
11 Les participants ont insisté avec force sur le fait qu'il serait illusoire de prétendre d'enrayer définitivement les "échanges occultes". Il faudrait plutôt, comme le suggère Mamadou Diouf, essayer de réguler la corruption. Autrement dit, il est question d'éviter qu'elle devienne débridée et de favoriser des formes de corruption "productives", à l'instar de quelques exemples asiatiques.

12 La piste, souvent suggérée dans les packages clefs en main de la bonne gouvernance, de formuler de nouveaux codes de conduite et de déontologie professionnelle, est séduisante mais sans futur, d'après les intervenants. La réalité est plutôt celle des déontologies bricolées, propres à des cultures professionnelles contextualisées. Une socialisation effective dans les normes déontologiques est sans doute indispensable, mais elle devrait aller de pair avec une amélioration significative de la situation économique du personnel administratif.

13 Il est aussi illusoire de penser que des réformes législatives ou institutionnelles suffiront à résoudre le problème, car beaucoup d'États africains, comme l'a suggéré Stephen Ellis au cours du débat, sont des États en trompe-l'œil, qui satisfont le public international en n'assurant qu'une apparence d'Etat de droit.

14 En revanche, on a souligné le rôle crucial que peut jouer le système judiciaire, qui n'est pas épargné, loin de là, par le phénomène qu'il est censé combattre. Du reste, il se trouve le plus souvent dans l'impossibilité de le faire, devant compter sur des magistratures politiquement soumises, voire complètement embrigadées par les pouvoirs exécutif et législatif.

Quelles voies de recours et quelles formes d'expression s'offrent alors aux usagers ? Les radios, plus que les journaux, semblent pouvoir offrir des lieux de discussion et de critique. Il faut toutefois considérer que les opinions publiques sont de plus en plus laissées par des campagnes de lutte sans résultats concrets.

\section{AUTEURS}

\section{GIORGIO BLUNDO}

Maître de conférence à l'EHESS, Centre de la Vieille Charité. 2. rue de la Charité 13002

Marseille, tel. : (+33) 0491140121 ; fax. : (+33) 0491913401 ;

blundo@ehess.cnrs-mrs.fr

\section{WALTER E.A. VAN BEEK}

African Studies Centre, Leiden, tel : (+31) 0715273363 ; w.vanbeek@fss.uu.nl 